\title{
Application of an Innovative Data Analytical Approach to Assessing the Disease Situation during Crises in Somalia, Yemen and Pakistan
}

\author{
Kamran Ahmed*, Antony Ajanga, Omer A. Saleh, Mohammad D. Altaf and Ghulam R. \\ Popal
}

World Health Organization EMRO, Nairobi, Kenya

\section{Objective}

To assess the epidemic and outbreak situations during emergencies through development and application of a data summarization techniques while launching electronic disease early warning systems (eDEWS) in resource poor countries

\section{Introduction}

Infectious disease outbreaks during crises can be controlled by detecting epidemics at their earliest possible stages through cost effective and time efficient data analytical approaches. The slow or non reporting is a real gap in existing reporting systems that delays in receiving the disease alerts and outbreaks, and hence delays in response causing high burden of morbidity and mortality, especially during crises situation. As on contrary, the functioning electronic databases for fast and reliable disease early warning and response networks (EWARN) have been found very effective in early detection, confirmation and response to disease outbreaks but launching the implementation of such systems is always time consuming due to resource constraints and other limitations during crises. Hence introduction of time efficient data analytical approaches can serve as a fast and reliable alternative for electronic databases during the launching phases, and may facilitate assessment of epidemics and outbreak situation by ensuring immediate, reliable and fully functional disease reporting and analysis until online database becomes fully functional and adopted by authorities.

\section{Methods}

Here, we describe an innovative and low-cost simple analytical method - called Data Summarization Technique (DST) - that was developed to assess the disease situation for early detection of epidemics while launching the implementation of online database system during humanitarian crises in resource poor countries. The DST approach is easily implementable in practice and has been developed on advanced time efficient Ms Excel pivot table technique that require basic Excel skills at end user level and is customizable for better integration with other databases (example Epi Info \& Access) of vertical health programmes in order to generate semi-automated epidemiological reports on timely and ad-hoc basis, an important approach to perform well during emergencies. Local capacity was built to utilize DST tools for complete data analysis using simulation exercises where basic Ms.Excel knowledge was considered essential for health staff participation in trainings.

\section{Results}

The development and implementation of eDEWS system took one month on average to become functional in conflict-hit regions and faced many challenges such as limited skilled human resource capacities and access (internet \& electricity issues), and other technical and financial considerations. The DST method was successfully tested as an alternative data analytical approach during initial phase of launching of eDEWS to keep monitoring disease situation until online database becomes fully functional. Health staff in all humanitarian settings found DST as a simple and flexible tool for data analysis and EpiBulletin generation as it requires minimum set of analytical skills, and also that it facilitates linking of various databases of vertical health programmes in order to generate disease situation updates for stakeholders. Its multilingual support also facilitated offline production of epidemiological bulletins in different languages at national and regional levels, and allowed rapid sharing of epidemiological information through official web pages of humanitarian agencies. Introduction of DST approach has also improved timeliness of sharing of information of public health importance from many weeks to few hours or a day in all three countries.

\section{Conclusions}

Our results indicate that DST is a very useful analytical approach to monitor health situation without any delay during humanitarian crises where poor capacity and issues with access and resources always cause delays in implementation of launching of electronic tools for disease early warning system. Furthermore, this innovative approach can help in improving overall public health information management capacities and capabilities of resource poor settings during emergencies due to its flexibility and simplicity in order to meet the challenges in absence of electronic automated analysis tools and with limited skilled human resource capacities.

\section{Keywords}

Early outbreak detection; Data Analysis; Humanitarian Crises; Electronic Database; Surveillance system

\section{Acknowledgments}

We wish to thank our data management and eDEWS surveillance teams, too numerous to list individually, who provided us with prompt help, information and facilitation.

\section{References}

1. Ahmed K, Altaf MD, Dureab F. Electronic infectious disease surveillance system during humanitarian crises in Yemen. OJPHI. 2014. 6(1).

\author{
*Kamran Ahmed \\ E-mail: drkamranrajput@hotmail.com
}

